\title{
Ballistic Target PHD Filter Based on Infrared Focal Plane Ambiguous Observation
}

\author{
Wei Zhao and Shucai Huang \\ Air and Missile Defense College, Air Force Engineering University, Xian, Shaanxi, China \\ Correspondence should be addressed to Wei Zhao; shnxshdny@163.com and Shucai Huang; hsc67118@126.com
}

Received 5 June 2017; Accepted 11 October 2017; Published 6 November 2017

Academic Editor: Vladimir Turetsky

Copyright ( 2017 Wei Zhao and Shucai Huang. This is an open access article distributed under the Creative Commons Attribution License, which permits unrestricted use, distribution, and reproduction in any medium, provided the original work is properly cited.

\begin{abstract}
Space-based early warning system, the main detection means of which is passive detection based on focal plane, is an important part of ballistic missile defense system. The focal plane is mainly composed of CCD, and its size can reach the micron level, so the pixel is often regarded as point of no area in image postprocessing. The design of traditional tracking methods is based on this, and the observation based on the focal plane is modeled as the azimuth with random noise. However, this modeling is inaccurate. In the context of space-based detection, CCD cannot be simplified as a point, and its size should be considered. And the corresponding observation cannot be treated as azimuth with random noise. In this paper, the observation based on focal plane is modeled as Unambiguously Generated Ambiguous (UGA) measurement. The PHD filter algorithm is redesigned and simplified. The simulation results show that the algorithm based on UGA measurement observation model has better tracking effect compared with that based on traditional observation model. This method provides technical support for more accurate target tracking for space-based early warning system.
\end{abstract}

\section{Introduction}

The world has always attached great importance to the development of space-based early warning system to deal with the threat of ballistic missile. The United States is the most advanced country that has carried out a lot of research work about MIDAS, DSP, SBIRS, and STSS $[1,2]$. DSP has been applied in Gulf War, which proved the strategic significance of space-based early warning system. In recent years, the detection mechanism extends from infrared to visible and ultraviolet, and the detection band also extends from a single band to multiple bands and to multispectral and hyperspectral $[3,4]$. Limited by technology, the size of CCD does not meet the actual detection needs [5-7], especially the need for spatial resolution, which will affect the accuracy of target tracking. There are many research works about detection and tracking capability of space-based early warning system and specific target detection and tracking algorithms $[8,9]$. After investigating a variety of detection and tracking algorithms based on space-based early warning system, it is found that the infrared passive detection is mostly used in which the direction of arrival (DOA) is detected. The specific data are the azimuth and pitch angle of the target relative to the sensor, and the detection data will also be analyzed in Section 2. Actually, the infrared detection is mostly based on the focal plane [10]; thus the data is recorded in the form of infrared image which describes the detected infrared radiation intensity of the target and background. The pixel is the smallest resolution treated as a point pointing to a precise direction in usual treatment. But this is not realistic and we could not obtain a precise direction from a pixel. In ordinary application the small pointing error may not have a significant impact, but it has a completely different effect in space-based detection. It is for this reason that accurate modeling of space-based infrared detection is proposed, and tracking filter based on this observation model is designed.

The observation error is analyzed in Section 2, and the observation based on focal plane imaging was modeled. In Section 3, the motion of target image on focal plane is studied, and the constant acceleration model is proved to be reasonable. In Section 4, the PHD filter algorithm based on UGA measurement observation modeling is given. In 


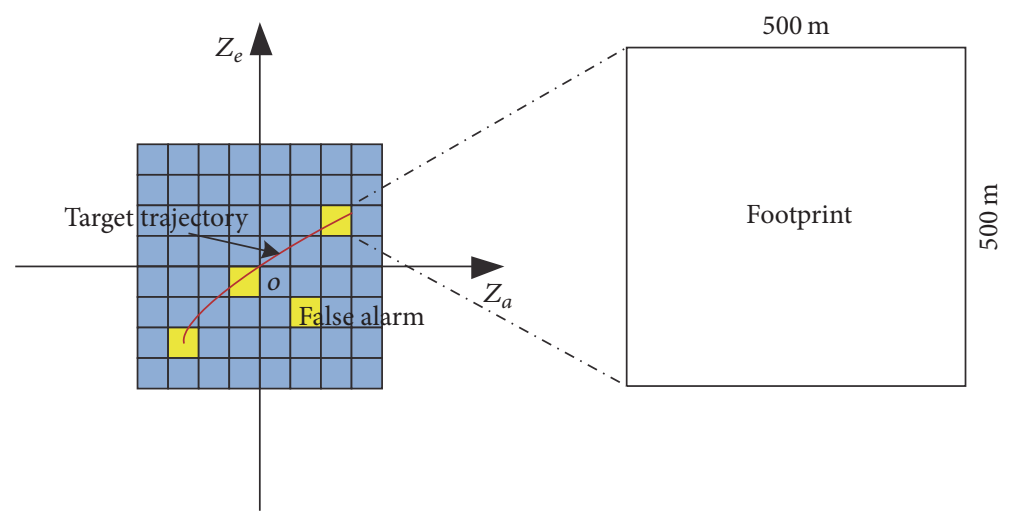

FIgURE 1: CCD and its footprint.

Section 5, the difficulty of implementing the filter algorithm is analyzed, and a simplified algorithm is given. In Section 6, the PHD filters based on traditional observation modeling and UGA measurement observation modeling are simulated and compared in the same scenario. And the conclusions are given in the last section.

\section{Traditional Observation and UGA Measurement Observation}

Passive observation refers to an observation method that only obtains the target's direction of arrival rather than its position. It is an important detection method in military application because of its good concealment and high angular resolution. Commonly used passive detection methods include infrared, ultraviolet, visible, electromagnetic wave, and sonar methods which determine the direction of the target relying on receiving the signal emitted or reflected by the target. Passive observation is often modeled as angle containing noise, just as it is shown in (1); this is a generic modeling method that applies to all of the above-mentioned detection methods.

$$
\mathbf{z}_{k+1}=\eta_{k+1}\left(\mathbf{x}_{k+1}\right)+\mathbf{V}_{k+1} \text {. }
$$

In traditional studies on target tracking based on space-based infrared detection, the observation data is concerned as angle containing noise too, which could be called point observation. In fact this is not true, because $\mathbf{z}_{k+1}$ could not be obtained accurately.

The orbit of satellite is so high that the detector can get a wide field of view. This is both its advantage and its disadvantage. Due to technical conditions, the infrared CCD cannot be small enough to meet the actual detection needs; what is more, the detector is so high, just as it is shown in Figure 1, where the detection area of a CCD can be tens of thousands of square meters. And this is the great difficulty of target positioning and tracking.

Therefore the observation based on focal plane is different from traditional passive observation; it belongs to Unambiguously Generated Ambiguous (UGA) measurement. The observer could know the CCD in whose field of view the target appears but could not get the accurate direction. UGA measurement observation has the following characteristics:
First, the observation modeling itself has a certain degree of ambiguity; second, the relationship between the observation and the target state can be described by an accurate sensor conversion model; for example, $\mathbf{z}=\eta(\mathbf{x})$.

In fact, the observation data obtained by focal plane imaging can be expressed accurately by pixels corresponding to CCDs whose signals exceed the threshold. Such observations can be called pixel observation, which could be denoted as $E_{z}$. The image of the target in the focal plane is in $E_{z}$ but could not be obtained accurately, limited to resolution. So this kind of observation is UGA measurement observation. The observation equation can be expressed as follows:

$$
E_{\mathbf{Z}_{k+1}}=E_{\eta_{k+1}\left(\mathbf{x}_{k+1}\right)+\mathbf{V}_{k+1}}
$$

It is assumed that random noise $\mathbf{V}_{k+1}$ denotes observation noise caused by detector position error and pointing error. $\mathbf{V}_{k+1}$ is white Gaussian noise, and its covariance is only related to the detector itself; $E\left(\mathbf{V}_{k+1} \cdot \mathbf{V}_{k+1}^{T}\right)=\mathbf{R}_{k+1}$.

Generalized observation is defined as $\boldsymbol{\Theta}_{\mathbf{z}_{k+1}} \triangleq E_{\mathbf{z}_{k+1}-\mathbf{V}_{k+1}}$, and the generalized likelihood function is shown as

$$
f\left(\boldsymbol{\Theta}_{\mathbf{z}_{k+1}} \mid \mathbf{x}_{k+1}\right) \triangleq \operatorname{Pr}\left(\eta\left(\mathbf{x}_{k+1}\right) \in \boldsymbol{\Theta}_{\mathbf{z}_{k+1}}\right) .
$$

\section{Target Motion Modeling}

To establish a reasonable target motion model, the dynamic model of ballistic target is established firstly. The acceleration, velocity, and trajectory in plane are shown in Figure 2 and the sketch map of focal plane imaging is shown in Figure 3. It is assumed that the target is launched from $\left(\mathrm{E} 0^{\circ}, \mathrm{N}^{\circ}\right)$, the direction is northeast $45^{\circ}$, and the detector is on the GEO satellite above that launch point. After calculation, we can get the image trajectory of the target on focal plane, just as shown in Figure 4.

The distance and height of target changing over time are shown in Figure 5. As shown in Figure 6, through the quadratic fitting of the distance versus time and height versus time, it is found that the fitting effect is good and the residual is small enough. And the quadratic polynomial corresponds to the constant acceleration (CA) model; hence the CA model 

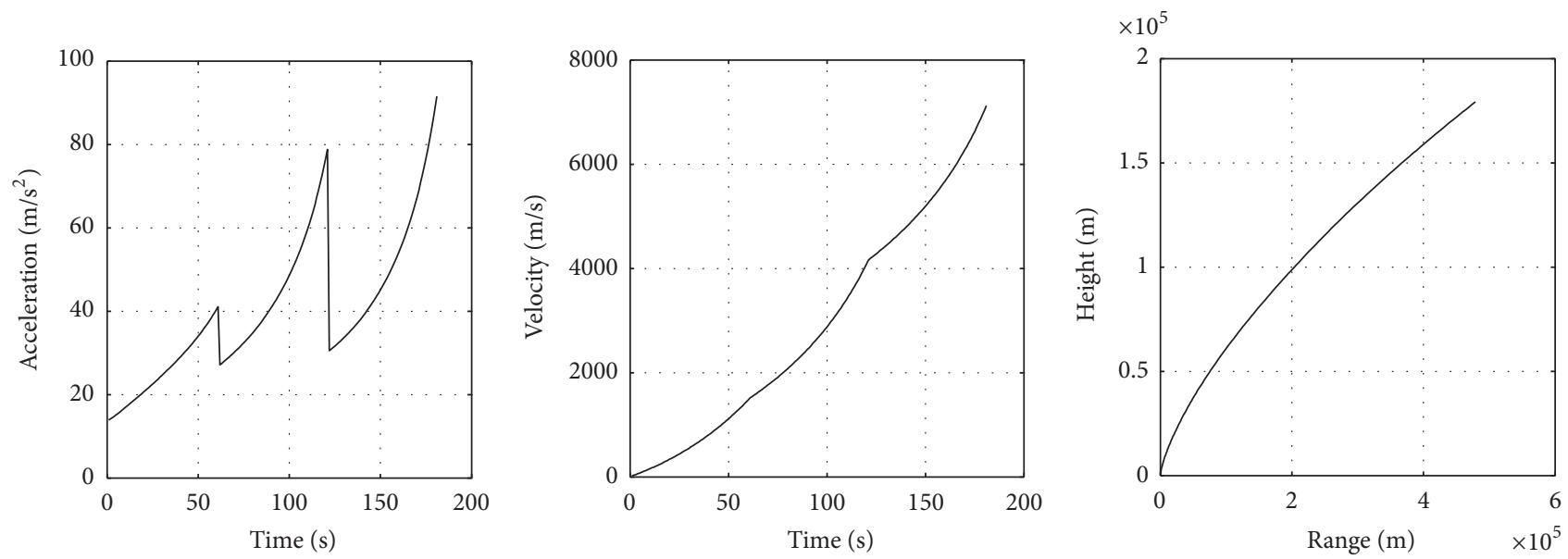

FIgURE 2: Acceleration velocity and trajectory of a target.

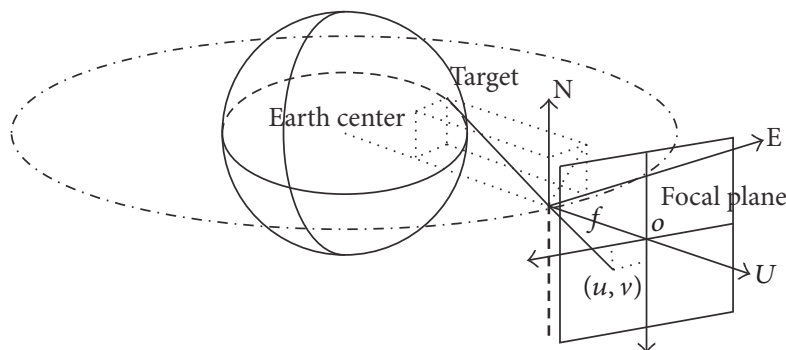

FIGURE 3: Sketch map of focal plane imaging.

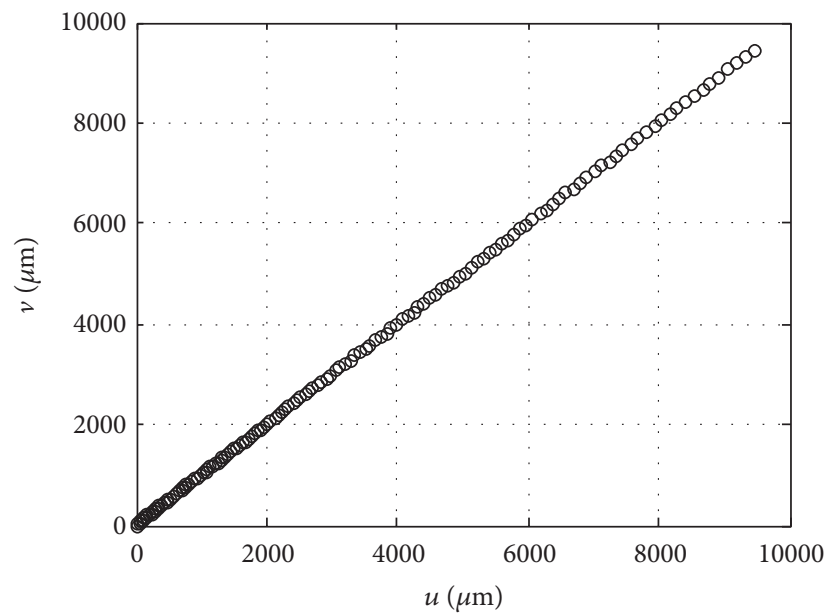

FIgURE 4: Trajectory of target image on focal plane.

is chosen as target motion model in this paper, just as it is shown in

$$
\left[\begin{array}{c}
x \\
\dot{x} \\
\ddot{x}
\end{array}\right]_{k+1}=\left[\begin{array}{ccc}
1 & \Delta & \frac{\Delta^{2}}{2} \\
0 & 1 & \Delta \\
0 & 0 & 1
\end{array}\right]\left[\begin{array}{c}
x \\
\dot{x} \\
\ddot{x}
\end{array}\right]_{k}+\mathbf{W}_{k+1} \text {, }
$$

where $\Delta$ denotes time step, $\mathbf{W}_{k+1}$ denotes process noise of target motion equation, and $E\left(\mathbf{W}_{k+1} \cdot \mathbf{W}_{k+1}^{T}\right)=\mathbf{Q}_{k+1}$.

\section{Filtering Algorithm}

In actual combat environment, multitarget tracking is unavoidable for space-based infrared early warning system. Therefore, PHD filter is chosen as the tracking algorithm in this paper. Firstly, PHD filter is introduced briefly.

The PHD filter is an advanced approximation technique for multitarget Bayesian filtering and consists of two parts: prediction and update.

It is known that probability hypothesis density is $D_{k \mid k}(\mathbf{x}) \stackrel{\text { abbr. }}{=} D_{k \mid k}\left(\mathbf{x} \mid \mathbf{Z}^{(k)}\right)$.

$$
\begin{aligned}
& \text { Prediction Equation. } D_{k+1 \mid k}(\mathbf{x}) \stackrel{\text { abbr. }}{=} D_{k+1 \mid k}\left(\mathbf{x} \mid \mathbf{Z}^{(k)}\right) \\
& \qquad \begin{aligned}
D_{k+1 \mid k}(\mathbf{x})= & b_{k+1 \mid k}(\mathbf{x}) \\
& +\int F_{k+1 \mid k}\left(\mathbf{x} \mid \mathbf{x}^{\prime}\right) \cdot D_{k \mid k}\left(\mathbf{x}^{\prime}\right) d \mathbf{x}^{\prime}
\end{aligned}
\end{aligned}
$$

where pseudo-Markov transition density is

$$
\begin{aligned}
F_{k+1 \mid k}\left(\mathbf{x} \mid \mathbf{x}^{\prime}\right) \triangleq & p_{s}\left(\mathbf{x}^{\prime}\right) \cdot f_{k+1 \mid k}\left(\mathbf{x} \mid \mathbf{x}^{\prime}\right) \\
& +b_{k+1 \mid k}\left(\mathbf{x} \mid \mathbf{x}^{\prime}\right) .
\end{aligned}
$$

Update Equation. $D_{k+1 \mid k+1}(\mathbf{x})=L_{\mathbf{Z}}(\mathbf{x}) \cdot D_{k+1 \mid k}(\mathbf{x})$

$$
\begin{aligned}
L_{\mathbf{Z}}(\mathbf{x}) \triangleq & 1-p_{D}(\mathbf{x}) \\
& +p_{D}(\mathbf{x}) \sum_{\mathbf{z} \in \mathbf{Z}} \frac{L_{\mathbf{z}}(\mathbf{x})}{\lambda c(\mathbf{z})+D_{k+1 \mid k}\left[p_{D} L_{\mathbf{z}}\right]} .
\end{aligned}
$$

Under the condition of UGA measurement observation modeling, likelihood function in the update equation should be changed to (8). And the filter schemes for different observation models are shown in Figure 7. 

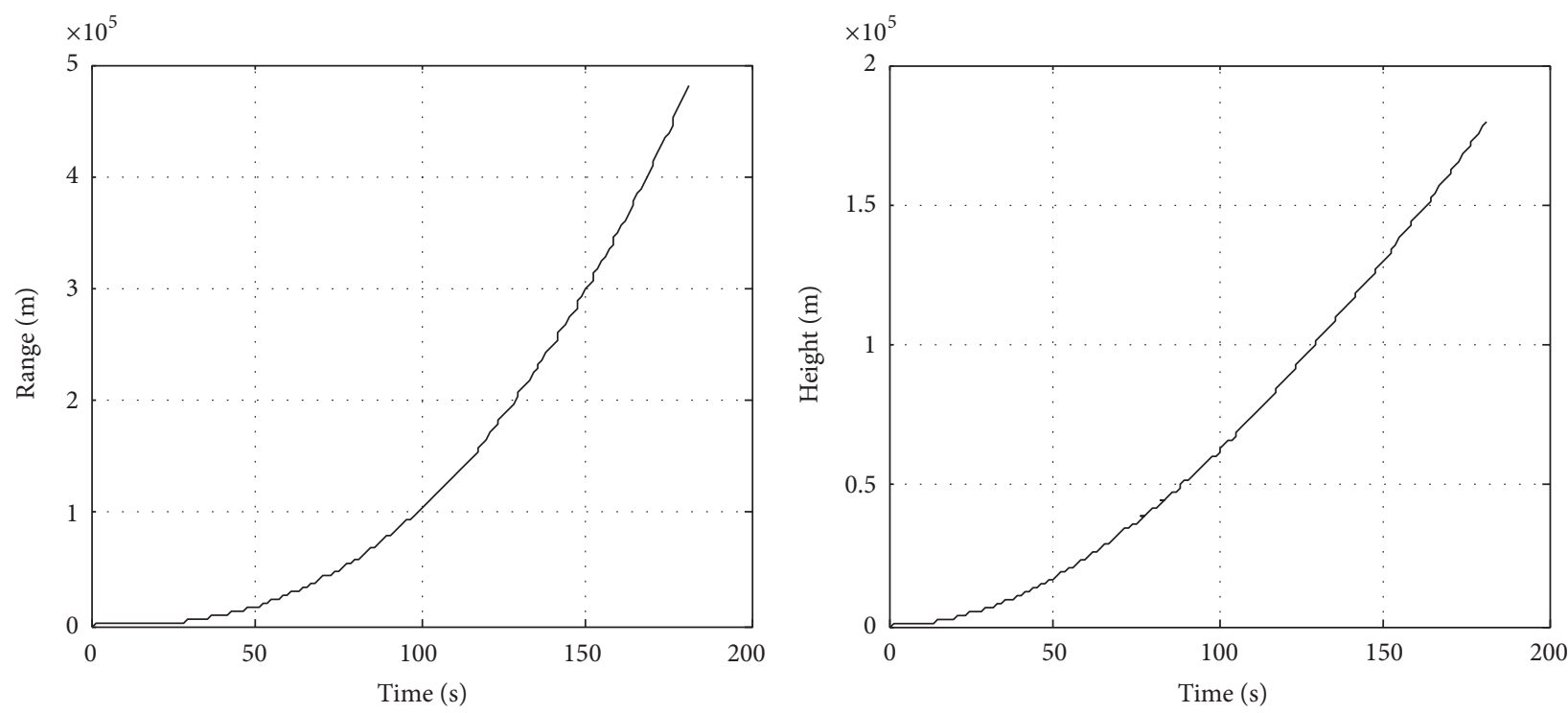

FIGURE 5: Range and height versus time.
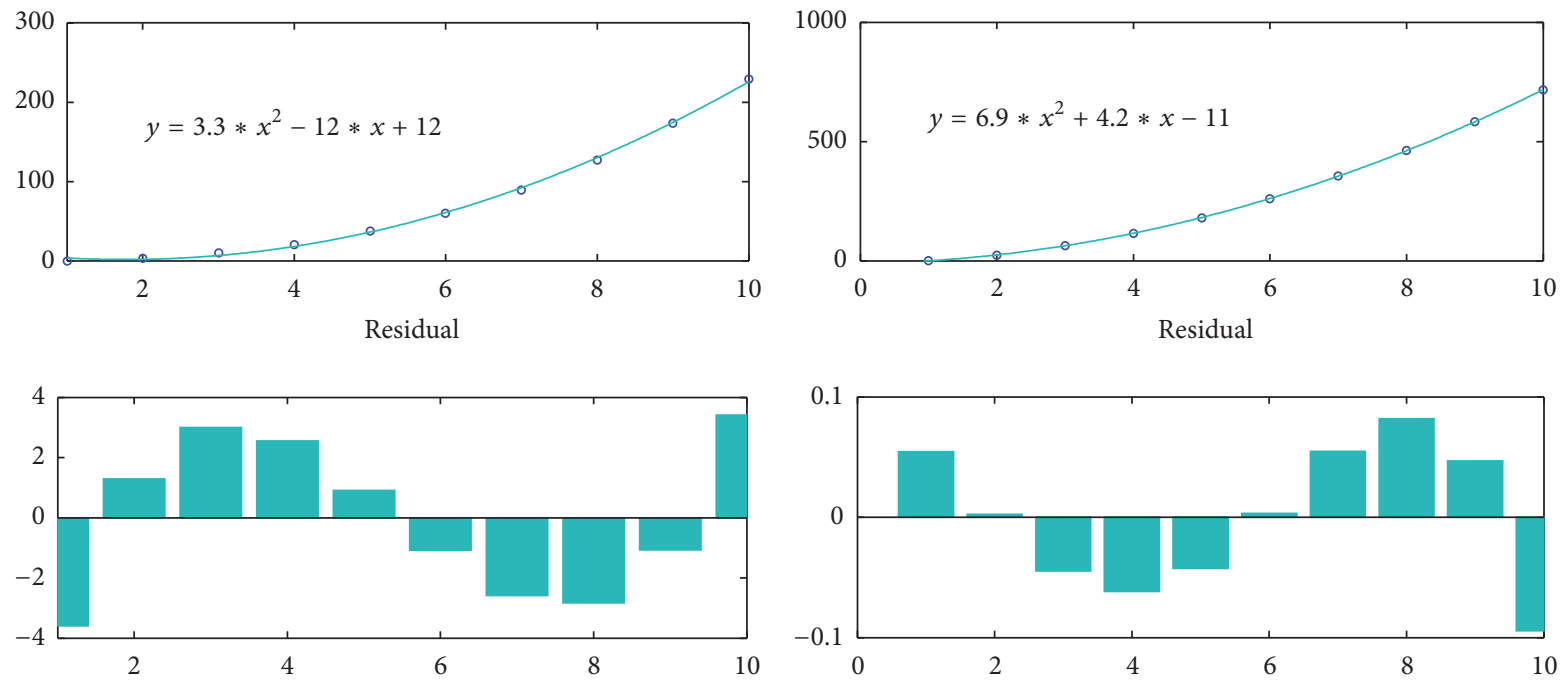

FIGURE 6: Range and height fitting and residual in 10 seconds.

In theory, $\operatorname{Pr}\left(\eta\left(\mathbf{x}_{k+1}\right) \in \boldsymbol{\Theta}\right)$ in (3) is obtained through integrating $\eta(\mathbf{x})$ over $\boldsymbol{\Theta}$.

$$
\operatorname{Pr}\left(\eta\left(\mathbf{x}_{k+1}\right) \in \boldsymbol{\Theta}_{\mathbf{z}_{k+1}}\right)=\int_{\Theta_{\mathbf{z}_{k+1}}} \Phi\left(\mathbf{z}_{k+1}\right) d \mathbf{z} .
$$

$\Phi(\mathbf{z})$ follows normal distribution in observation space, the mean is $\eta(\mathbf{x})$, and the covariance is $\mathbf{V}_{k+1}$.

\section{Simplification of Generalized Likelihood Function}

As described in previous section, in update step, the integration of normal distribution over generalized observation space needs to be calculated. But the integral complexity is not conductive to the implementation of the filter algorithm. Proper simplification is necessary.
A physical explanation of the PHD filter is given in literature [11], and another expression of update equation is given as shown in

$$
\begin{aligned}
f\left(\mathbf{Z}_{k+1} \mid \mathbf{c} \in \mathbf{\Xi}, \mathbf{Z}^{(k)}\right)= & p_{D}(\mathbf{c}) \cdot f\left(\mathbf{Z}_{k+1} \mid \mathbf{Z}^{(k)}\right) \\
& \cdot \frac{1}{\frac{1}{\mu} \sum_{j=1}^{m} \frac{L_{\mathbf{z}_{j}}(\mathbf{c})}{f\left(\mathbf{z}_{j} \mid \mathbf{Z}^{(k)}\right)}} \\
& +\left(1-p_{D}(\mathbf{c})\right) \\
& \cdot f\left(\mathbf{Z}_{k+1} \mid \mathbf{Z}^{(k)}\right),
\end{aligned}
$$

where $\mathbf{z}_{j}$ denotes the $j$ th pixel observation and $\mathbf{c}$ denotes state cell. 


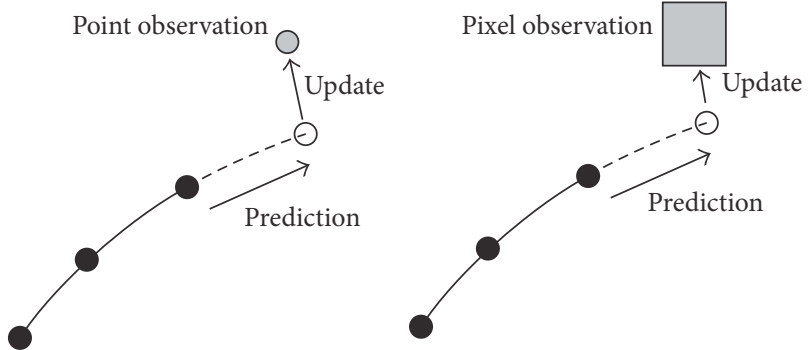

Figure 7: The filter schemes for different observation models.

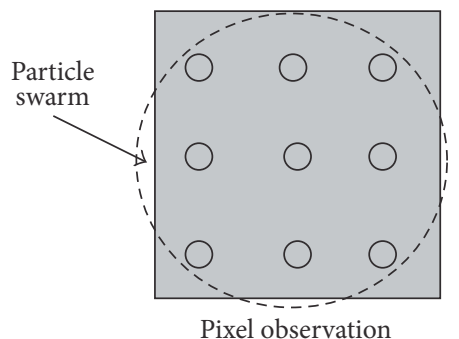

FIGURE 8: Sampling particles in image element.

The box part in (9) corresponds to the summation part of generalized likelihood function in (7). It can be seen that this part describes the ratio of the single-target likelihood function to the spatial density of the observation process, that is, the ratio of probability of $\mathbf{z}_{j}$ origins from state cell $\mathbf{c}$ to probability of $\mathbf{z}_{j}$ origins from others state cells and false alarms. Therefore, it is appropriate to choose a method to replace the ratio of probability here.

In this paper, the particle swarm is chosen to replace the pixel observation to describe its relationship with the prediction observation. As shown in Figure 8, nine particles are sampled in the pixel, and the sampling criterion is that the particles are evenly distributed in the pixel. The number of particles is denoted by $v$, and the particle is denoted as $p_{i}^{j}(i=1 \cdots v)$, where $i$ denotes the $i$ th particle. The likelihood function of particle and prediction observation can be expressed as

$$
\begin{aligned}
& L_{\mathbf{p}_{i}^{j}}\left(\eta\left(\mathbf{x}_{k+1 \mid k}\right)\right)=\frac{1}{2 \pi|\mathbf{V}|^{1 / 2}} \\
& \quad \cdot \exp \left(-\frac{1}{2}\left(\mathbf{p}_{i}^{j}-\eta\left(\mathbf{x}_{k+1 \mid k}\right)\right)^{\tau} \mathbf{V}^{-1}\left(\mathbf{p}_{i}^{j}-\eta\left(\mathbf{x}_{k+1 \mid k}\right)\right)\right) .
\end{aligned}
$$

Finally, the pseudo-likelihood function between the pixel observation and the prediction observation can be obtained by summation.

$$
L_{\boldsymbol{\Phi}_{z_{k+1}}}\left(\eta\left(\mathbf{x}_{k+1 \mid k}\right)\right)=\sum_{i=1}^{v} L_{\mathbf{p}_{i}^{j}}\left(\eta\left(\mathbf{x}_{k+1 \mid k}\right)\right) .
$$

\section{Simulation and Comparison}

To verify the proposed PHD filter based on pixel observation or UGA measurement observation, the following simulation
TABLE 1: Launch point, direction, and time of three targets.

\begin{tabular}{lccc}
\hline & Target 1 & Target 2 & Target 3 \\
\hline Launch point & $\left(\mathrm{W} 5^{\circ}, \mathrm{N} 5^{\circ}\right)$ & $\left(\mathrm{E} 5^{\circ}, \mathrm{N} 5^{\circ}\right)$ & $\left(\mathrm{E} 5^{\circ}, \mathrm{S} 5^{\circ}\right)$ \\
Direction & $135^{\circ}$ & $225^{\circ}$ & $315^{\circ}$ \\
Discovery time & $t_{0}$ & $t_{0}+10 \mathrm{~s}$ & $t_{0}+20 \mathrm{~s}$ \\
\hline
\end{tabular}

TABLE 2: OSPA and runtime.

\begin{tabular}{lcc}
\hline & Point observation & Pixel observation \\
\hline OSPA & 30.5 & 22.5 \\
Runtime & $1.048 \mathrm{~s}$ & $3.282 \mathrm{~s}$ \\
\hline
\end{tabular}

scenario is designed. The PHD filter based on UGA measurement observation is compared with traditional image target tracking filter. The OSPA is chosen as the index to evaluate the performance of multitarget tracking, and the GM-PHD filter is used.

Scenario. It is assumed that the earth geosynchronous orbit satellite is equipped with infrared detector. The subastral point is located at $\left(\mathrm{E}^{\circ}, \mathrm{N} 0^{\circ}\right)$, and the focal plane of the detector is area array of $1024 \times 1024$ infrared CCD. The size of every CCD is $30 \mu \mathrm{m} \times 30 \mu \mathrm{m}$, and the frame rate is $1 \mathrm{~Hz}$. The LOS random error follows Gaussian distribution, the mean is zero, and derivation is $20 \mu \mathrm{rad}$. From time $t_{0}$, three ballistic targets appear in the field of view and are recorded by focal plane. The launch parameters of these three targets are shown in Table 1.

As shown in Figure 9, three targets are denoted by T1, T2, and T3, respectively, and their starting points are indicated by "O." The trajectories in Figure 9 are projection of the three ballistic targets on the focal plane. The movements of the three targets are affected by the thrust of the engine, the gravity of the earth, and the aerodynamics. So their movement on the focal plane is a complex movement. And the transition from 3-dimensional space to focal plane is shown in Figure 3. Through the study of the target image trajectory, taking into account the computational complexity, the motion of the target image can be modeled as the constant acceleration model with Gaussian noise.

In fact, the sensor is often affected by clutter and noise, resulting in some false alarms. Therefore, the false alarms are considered in the simulation scenario. The number of false alarms obeys Poisson distribution with a parameter of 5 , and they are evenly distributed throughout the field of view.

Figure 10 shows the false alarms on the focal plane in this scenario. The logos are enlarged for easy display.

The tracking effects of PHD filters based on two kinds of observation models are shown in Figure 11. It is not easy to see difference between these two figures, so OSPA of these two multitarget tracking filters are given in Figure 12, and the statistical results of OSPA and runtime of two filters are shown in Table 2. 


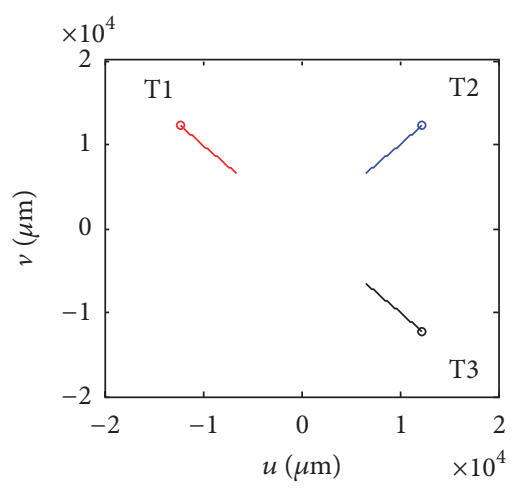

(a)

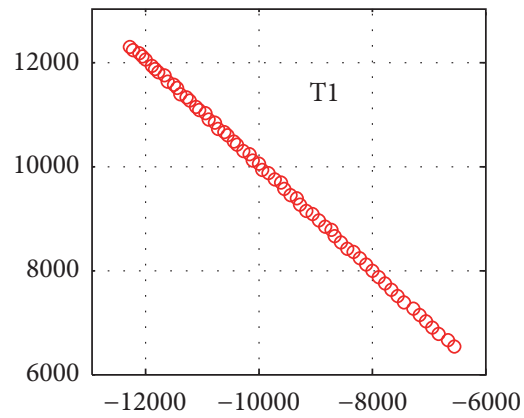

(b)

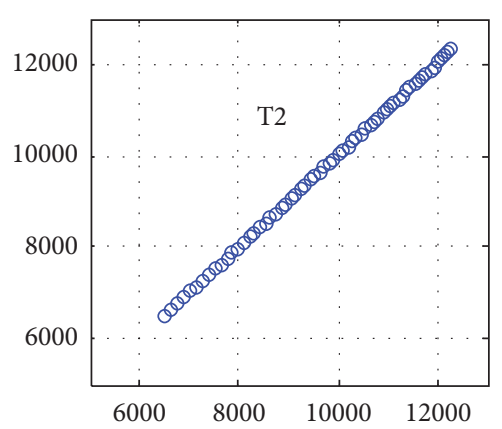

(c)

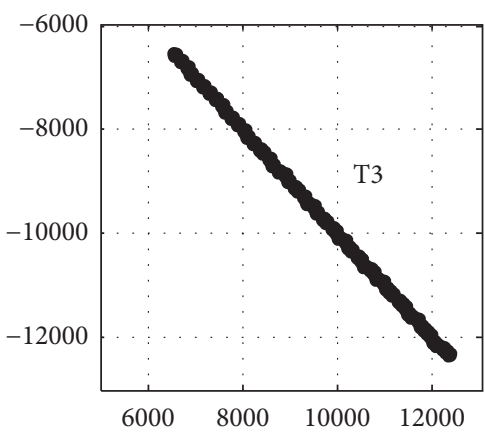

(d)

FIGURE 9: Target image trajectories on focal plane.

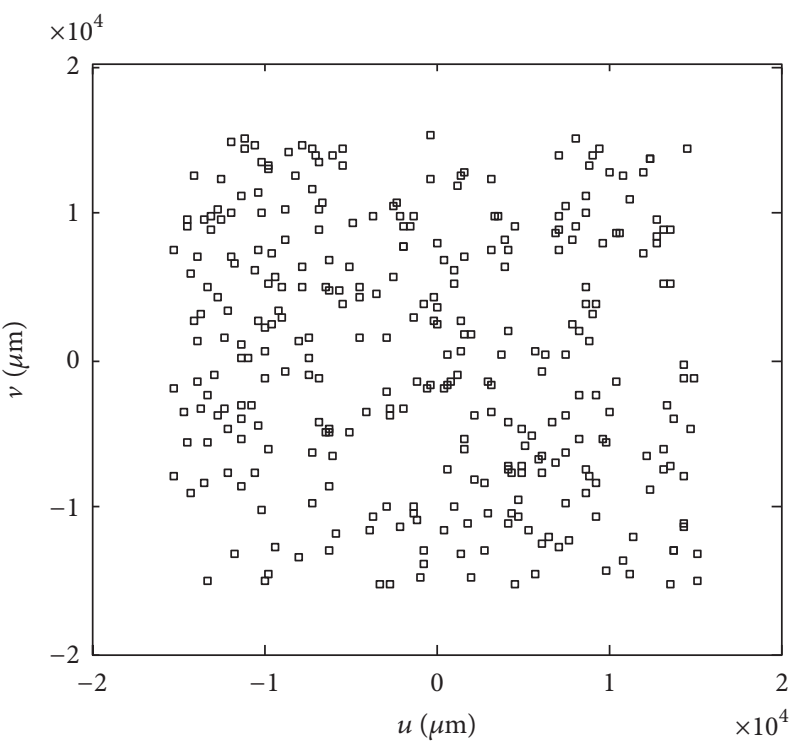

FIGURE 10: False alarms on focal plane.

Running Environment. The running environment is as follows: Intel ${ }^{\circledR} \operatorname{Core}^{\mathrm{TM}}$ i3-2120 CPU @ $3.30 \mathrm{GHz} 3.29 \mathrm{GHz}$, 3.24 GB RAM.

\section{Running Platform. The running platform is MATLAB R2014a.}

It is easy to see that the PHD filter based on pixel observation can obtain a more accurate target trajectory. And this is of great importance for ballistic target tracking based on space-based infrared focal plane detection and a breakthrough in traditional deterministic observation.

Obviously, the running time is closely related to the number of particles sampled from the pixel observation, and the specific statistical results are shown in Figure 13.

It is easy to see that the running time increases linearly with the increase in the number of particles. OSPA continues to decline as the number of particles increases; however, when the number of particles increases to 5, OSPA's decline 


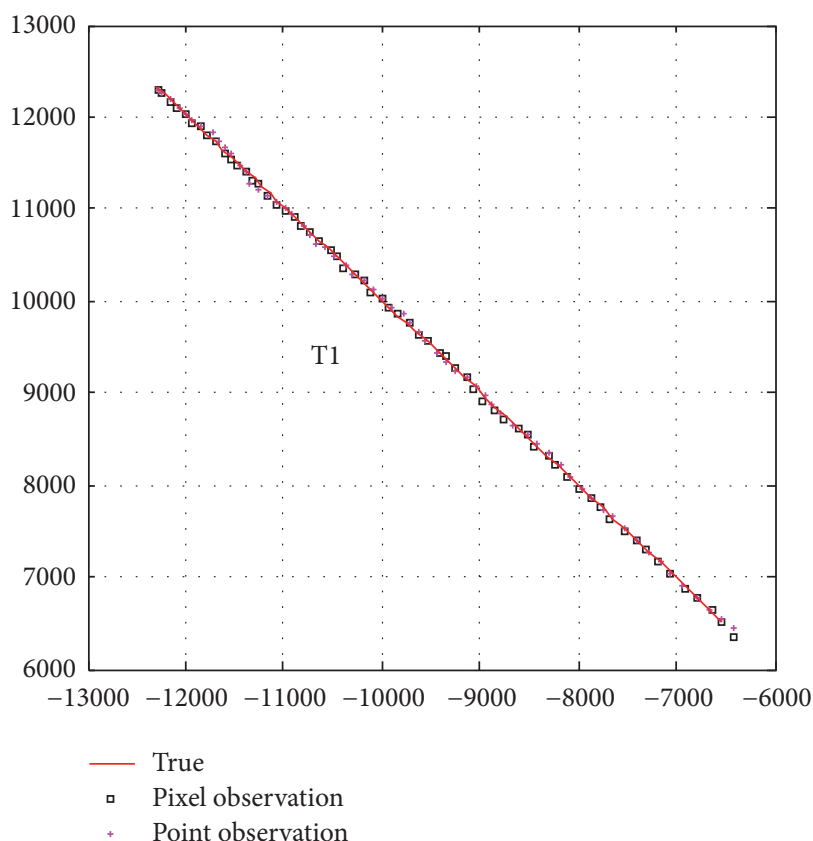

(a)

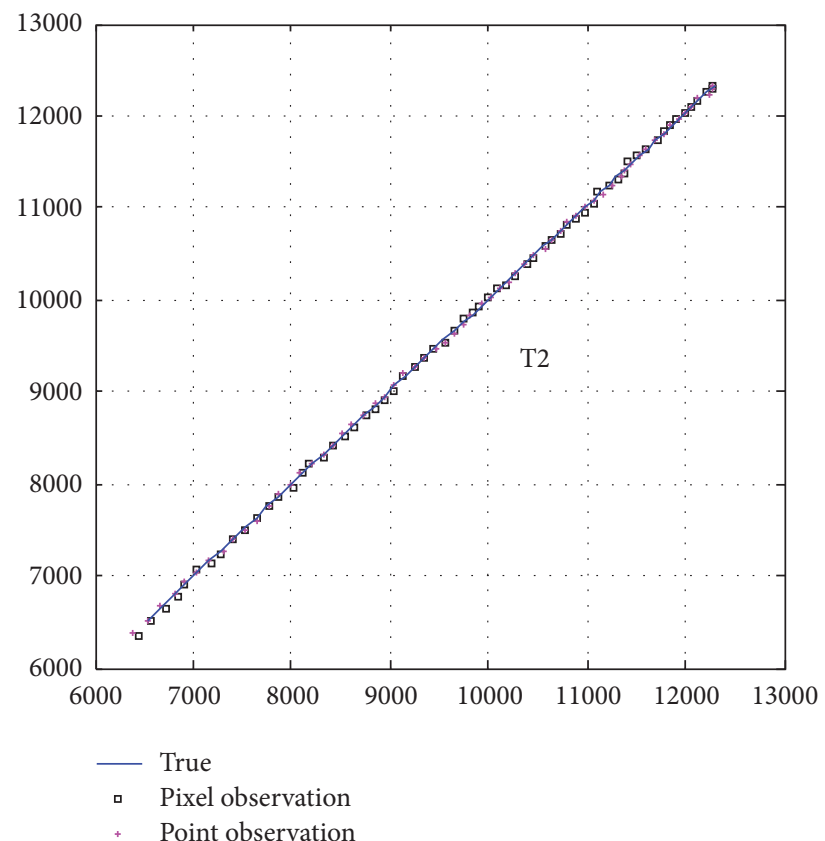

(b)

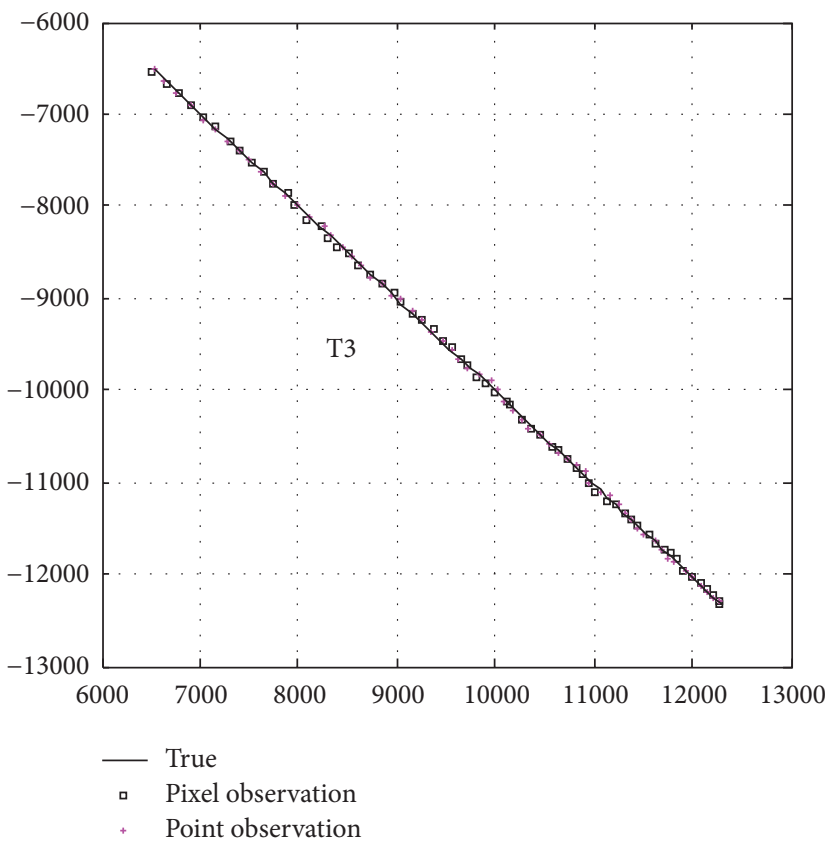

(c)

FIGURE 11: Estimated and true trajectories of three targets: (a) is target 1, (b) is target 2, and (c) is target 3.

has become very slow. Therefore, the number of particles used to replace the pixel observation needs to be chosen reasonably for different situations to meet certain accuracy.

\section{Conclusions}

The UGA measurement observation model based on focal plane imaging is studied. A more accurate observation model was established, and PHD filter algorithm based on pixel observation is given. Simulation results verify the accuracy of the observation modeling, and more accurate tracking is achieved. Unfortunately, the computation spent in the implementation of PHD filter based on pixel observation is several times more than that based on point observation. And the computation is closely related to the number of particles used in the simplification of the generalized likelihood function. To obtain better computation efficiency, we will continue to study the simplification of UGA measurement observation integral method. 


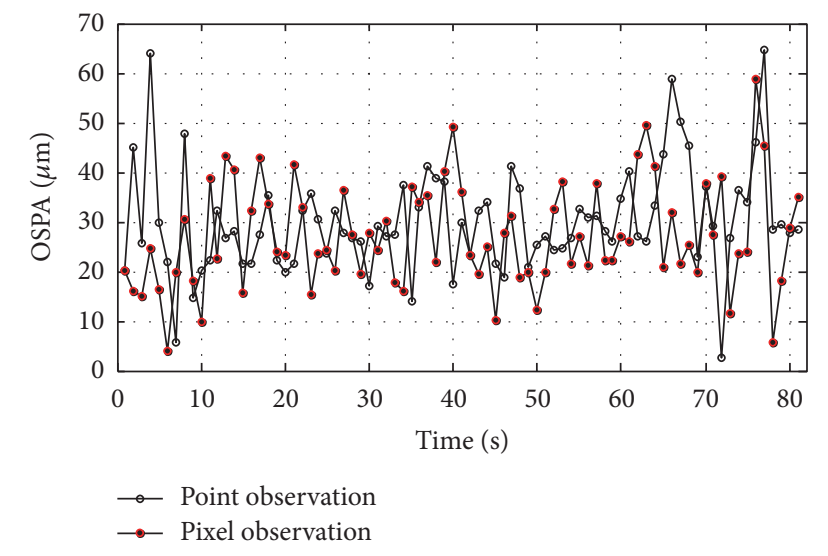

FIGURE 12: OSPA based on point observation and pixel observation.

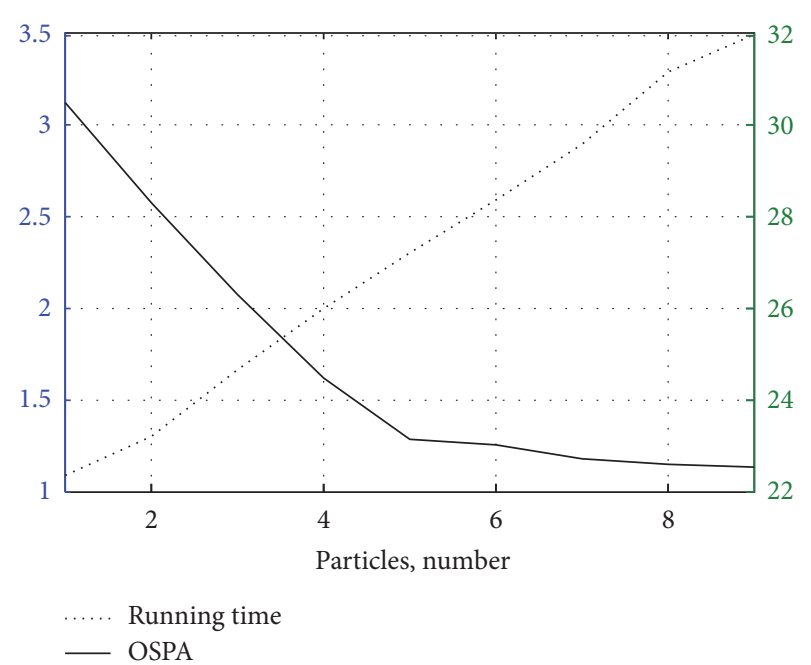

FIGURE 13: Running time and OSPA with different number of particles.

\section{Conflicts of Interest}

The authors declare that they have no conflicts of interest.

\section{References}

[1] J. Slattery and P. Cooley, "Space-based infrared satellite system (SBIRS) requirements management," in Proceedings of the 1998 IEEE Aerospace Conference, pp. 223-232.

[2] N. Andreas, "Space-Based Infrared System (SBIRS) system of systems," in Proceedings of the 1997 IEEE Aerospace Conference, pp. 429-438.

[3] H. F. Nelson, "Influence of participates on infrared emission from tactical rocket exhausts," Journal of Spacecraft and Rockets, vol. 21, no. 5, pp. 425-432, 1984.

[4] G. Avital, Y. Cohen, L. Gamss et al., "Experimental and Computational Study of Infrared Emission from Underexpanded Rocket Exhaust Plumes," Journal of Thermophysics and Heat Transfer, vol. 15, no. 1-4, pp. 377-383, 2001.

[5] S. Cheng, H. Zhang, H. Liu et al., "Performance measurement of mechanical shutter and dark current for scientific-grade optical
CCD," Guangxue Xuebao/Acta Optica Sinica, vol. 32, no. 2, Article ID 0204001, 2012.

[6] M. Z. Tidrow and W. R. Dyer, "Infrared sensors for ballistic missile defense," Infrared Physics \& Technology, vol. 42, no. 3, pp. 333-336, 2001.

[7] D. G. Oconnell and K. C. K. Cheung, "Multi-target-tracking optical sensor-array technology," in Proceedings of the SPIE, Sensors, Systems and Next-Generation Satellites XII, 2008.

[8] A. Benavoli, L. Chisci, and A. Farina, "Tracking of a ballistic missile with a-priori information," IEEE Transactions on Aerospace and Electronic Systems, vol. 43, no. 3, pp. 1000-1016, 2007.

[9] T. Shima, Y. Oshman, and J. Shinar, "Efficient multiple model adaptive estimation in ballistic missile interception scenarios," Journal of Guidance, Control, and Dynamics, vol. 25, no. 4, pp. 667-675, 2002.

[10] S. Tang, Research on satellite sensor resources management and detection tasks scheduling problem for LEO constellation of space-based early warning system, Graduate School of National University of Defense Technology Changsha, Hunan, China, 2011.

[11] P. S. Ronald and Mahler, Statistical Multisource-Multitarget Information Fusion, Artech House, London, UK, 2007. 


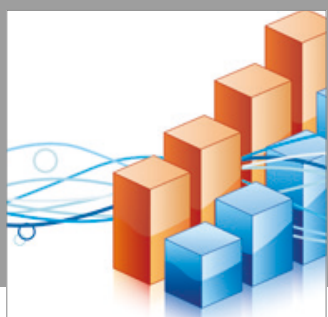

Advances in

Operations Research

vatersals

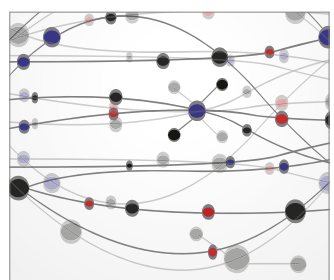

\section{The Scientific} World Journal
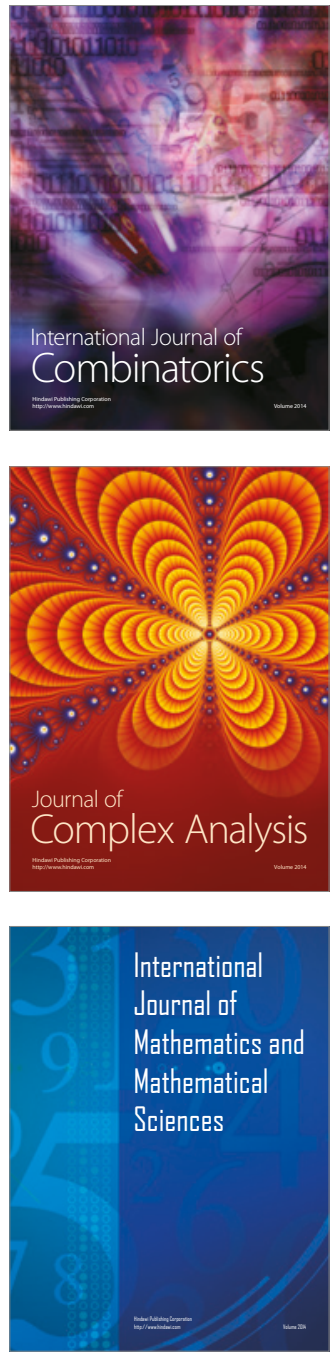
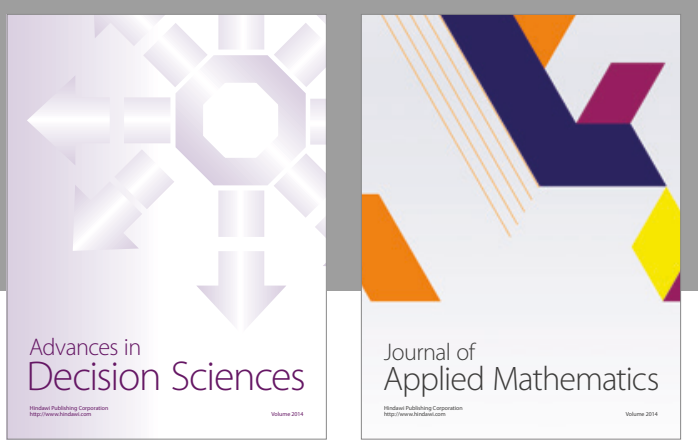

Algebra

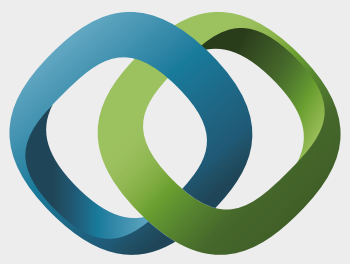

\section{Hindawi}

Submit your manuscripts at

https://www.hindawi.com
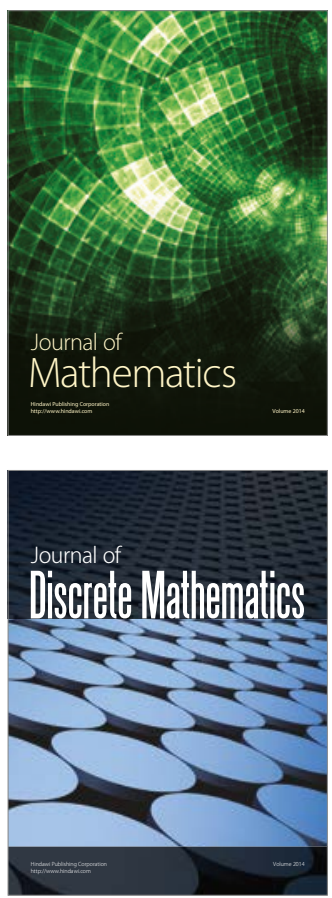

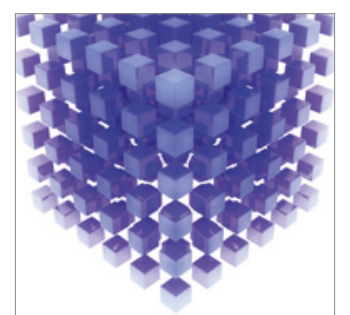

Mathematical Problems in Engineering
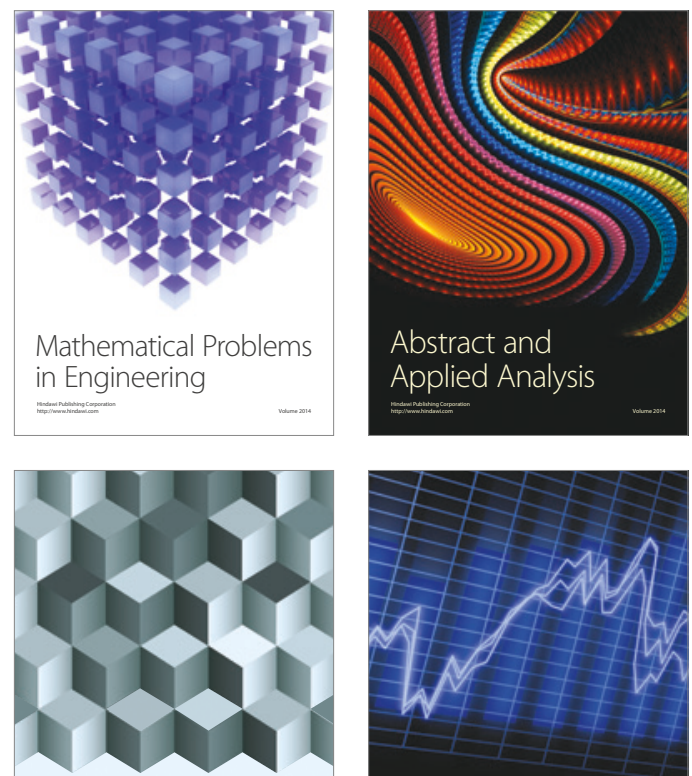

Journal of

Function Spaces

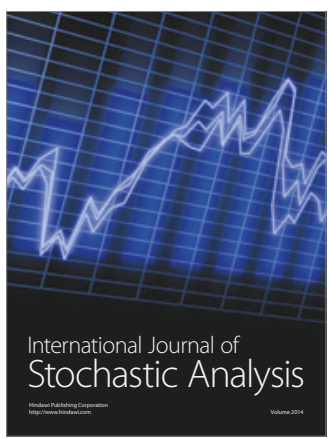

Probability and Statistics
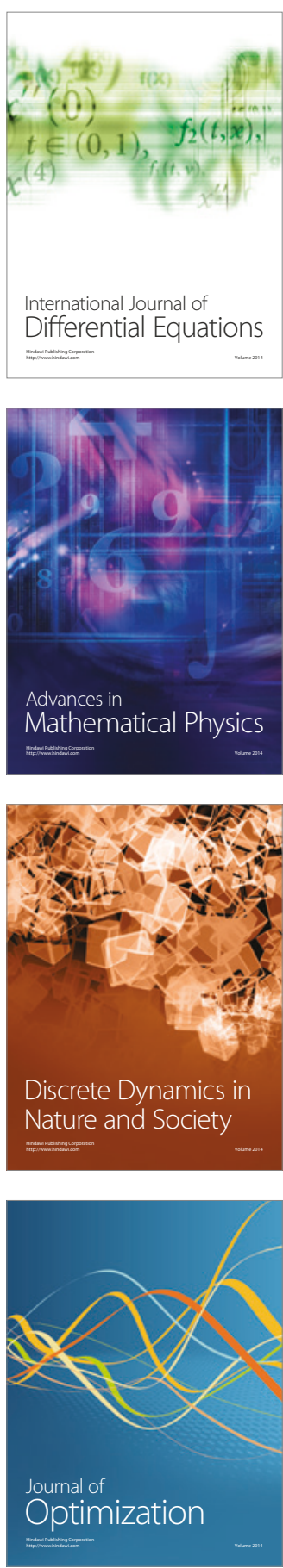